\title{
TEM Study of Bulk AIN Growth by Physical Vapor Transport
}

\author{
W.L. Sarney ${ }^{1}$, L. Salamanca-Riba ${ }^{1}$, T. Hossain ${ }^{2}$, P. Zhou ${ }^{2}$, H.N. Jayatirtha ${ }^{2}$, H.H. Kang ${ }^{1}$, \\ R.D. Vispute ${ }^{1}$, M. Spencer ${ }^{2}$, K.A. Jones ${ }^{3}$ \\ ${ }^{1}$ Dept. of Materials \& Nuclear Engineering, University of Maryland, College Park, MD, \\ ${ }^{2}$ Materials Science Research Center of Excellence, Howard University, Washington, \\ D.C., \\ ${ }^{3}$ U.S. Army Research Laboratory, Adelphi, MD
}

\begin{abstract}
We are attempting to grow bulk AlN that would be suitable as a substrate for nitride film growth. Bulk AlN films were grown by physical vapor transport on $3.5^{\circ}$ offaxis and on-axis $6 \mathrm{H} \mathrm{SiC} \mathrm{seed} \mathrm{crystals} \mathrm{and} \mathrm{characterized} \mathrm{by} \mathrm{TEM,} \mathrm{x-ray-diffraction,}$ Auger electron microscopy, and SEM. TEM images show that the bulk AlN does not have the columnar structure typically seen in AlN films grown by MOCVD. Although further optimization is required before the bulk AlN is suitable as a substrate, we find that the structural characteristics achieved thus far indicate that quality bulk AlN substrates may be obtained in the future.
\end{abstract}

\section{INTRODUCTION}

Despite the rapid progress made in nitride based semiconductor film growth, there are still no ideal substrates for high quality epitaxial growth. AIN and GaN are typically grown on $\mathrm{SiC}$, which is expensive, or on Sapphire, which is cheaper but has a large lattice mismatch with the nitride based films. Minimizing the lattice mismatch and enhancing the growth conditions improves the nitride film morphology by lowering the density of defects such as misfit dislocations. Other defects, such as inversion domain boundaries, result from the film's non-isomorphism with the substrate rather than the lattice mismatch [1]. The ideal substrate needs to be as lattice matched and isomorphic to the film as possible. Nitride buffer layers are often grown on sapphire or $\mathrm{SiC}$ in an attempt to provide a lattice matched and isomorphic surface for film growth. While buffer layers have helped improve film quality, films grown on buffer layers are usually columnar and contain a higher density of dislocations than desired. AlN or GaN substrates are necessary to achieve further film quality improvement. Due to the high melting temperatures and high dissociation pressures of III-N compounds, bulk nitride crystal growth is difficult. Although the exact nitrogen dissociation pressure is not known, values cited by Landolt and Börnstein [2] indicate that the nitrogen dissociation pressure of AlN is orders of magnitude smaller than that of GaN or InN [3]. Bulk AlN, therefore, should be easier to grow than bulk GaN or InN. In this study we examine the structural quality of bulk AlN grown by physical vapor transport and compare it to AlN buffer layers grown by MOCVD.

\section{EXPERIMENT}

Bulk AlN was grown by physical vapor transport by the decomposition of AlN powder in the presence of ambient nitrogen. The growth temperature range was $\mathbf{2 1 5 0}^{\circ}$ - 
$2200^{\circ} \mathrm{C}$ with nitrogen pressures of 400 410 Torr. The separation between the seed and AlN powder was approximately $4 \mathrm{~mm}$ under a temperature gradient of $1-3{ }^{\circ} \mathrm{C} / \mathrm{mm}$. The growth rate was varied between 10 and 50 microns per hour. We investigated two different seed crystals, including singular $6 \mathrm{H} \mathrm{SiC}$ and off-axis $6 \mathrm{H} \mathrm{SiC} \mathrm{miscut} 3.5^{\circ}$ toward the $[01 \overline{1} 0]$ direction.

This study focuses on the transmission electron microscopy (TEM) characterization of bulk AlN. We used a JEOL 4000FX TEM operated at $300 \mathrm{kV}$. Cross-sectional TEM samples were prepared using tripod polishing and room temperature ion milling. Further characterization was conducting with Auger electron microscopy, x-ray diffraction, and scanning electron microscopy.

\section{RESULTS \& DISCUSSION}

TEM images of the bulk AlN grown on $3.5^{\circ}$ off-axis and on on-axis $6 \mathrm{H} \mathrm{SiC}$ are shown in Figs. 1 and 2, respectively. As seen in Fig. 1, the substrate appears to be off axis by a much larger angle than the expected $3.5^{\circ}$. The bulk AlN in this sample was grown at a temperature of $2150^{\circ} \mathrm{C}$. Exposing the $\mathrm{SiC}$ to such a high temperature may have caused the steps to move and bunch together. Figures 1(a) and 1(b) are dark field images taken with (0002) and $(01 \overline{1} 0)$ two beam conditions, respectively. The bulk AIN is noncolumnar, as shown in Figs 1(a) and 1(b). The bands present near the substrate step suggest that there are also steps along the beam direction, normal to the page. There are several small defects imbedded in the AlN, one of which has been magnified and is shown in Fig. 1(d). The white contrast surrounding this defect may be a strain field. Although we do not currently know what these defects are, one possibility is that they are small oxygen or aluminum precipitates. Despite the step bunching of the substrate's surface, the high-resolution image (Fig. 1c) shows that the quality of the AlN/SiC interface is good and that the AlN is of good structural quality. Diffraction patterns, not shown here, indicate that the AlN is well-aligned with the substrate.

The bulk AlN grown on on-axis $\mathrm{SiC}$ is also non-columnar, as shown in the (0002) and $(01 \overline{1} 0)$ DF images (Figs. 2a and 2b). Several small defects are imbedded at or just above the AlN/SiC interface, one of which is magnified and shown in Fig. 2(d). It is not clear whether these defects are of the same nature as those seen in the AlN grown on the off-axis $\mathrm{SiC}$ seed. These defects are approximately ten times smaller than similar looking defects in the AlN grown on off-axis SiC. Their location near the substrate suggest that they could be small precipitates of $\mathrm{Si}$ or $\mathrm{C}$. Other possibilities include oxygen or aluminum precipitates, or dislocation loops. We did not see these defects in the highresolution image shown in Fig. 2(c). This may be because they are too far apart and the small area examined in high resolution did not include any of these defects, or because these defects have no contrast at high magnifications. Further investigation is necessary to determine the nature and composition of these defects. Figure 2(c) shows the high quality of the AlN/SiC interface and the structure of the bulk AlN.

Auger electron microscopy was used to detect possible incorporation of impurities such as oxygen or carbon within the AlN. Ion beam sputtering caused the sample surfaces to become completely charged, making it impossible to obtain a spectrum. If the sample contained carbon or oxygen impurities, the charge would dissipate more easily and a spectrum would be obtainable. We obtained a spectrum with other lower-quality AIN samples, which are not presented in this paper. The spectrum for the low-quality AIN 


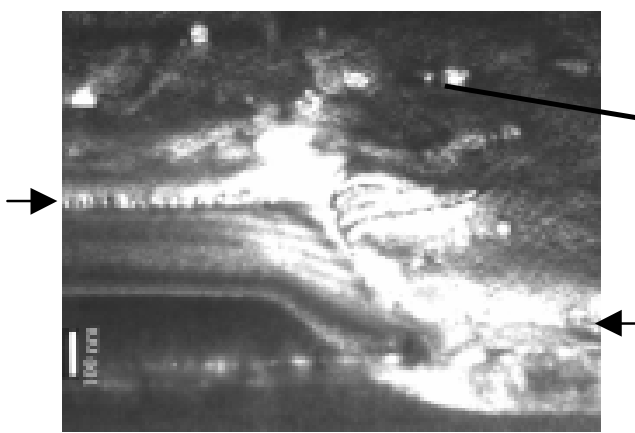

a

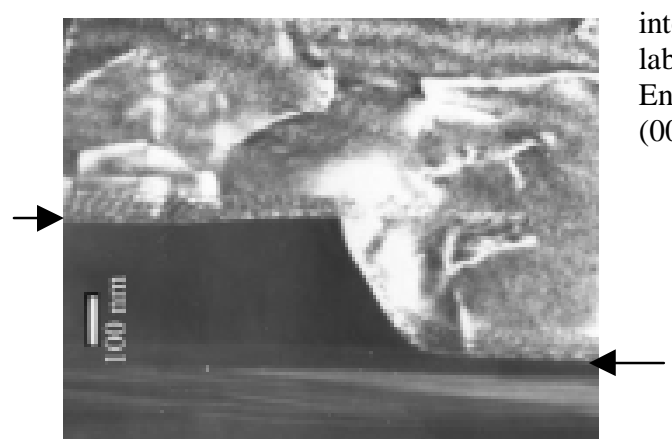

b

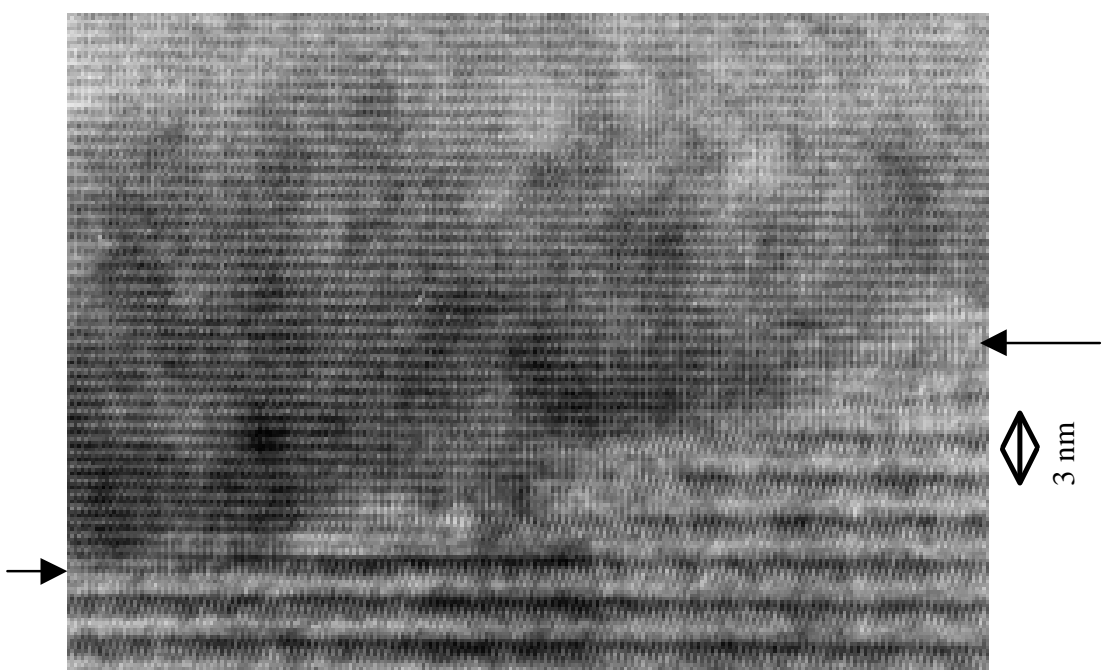

Fig. 1.(a) (0002) DF, (b) (01 $\overline{1} 0) \mathrm{DF}$ and $(\mathrm{c})$ high resolution lattice image of the bulk AlN/off-axis SiC

interface. The AlN/SiC interface is

labeled with the arrows. (d)

Enlargement of defect seen in the (0002) DF image. 


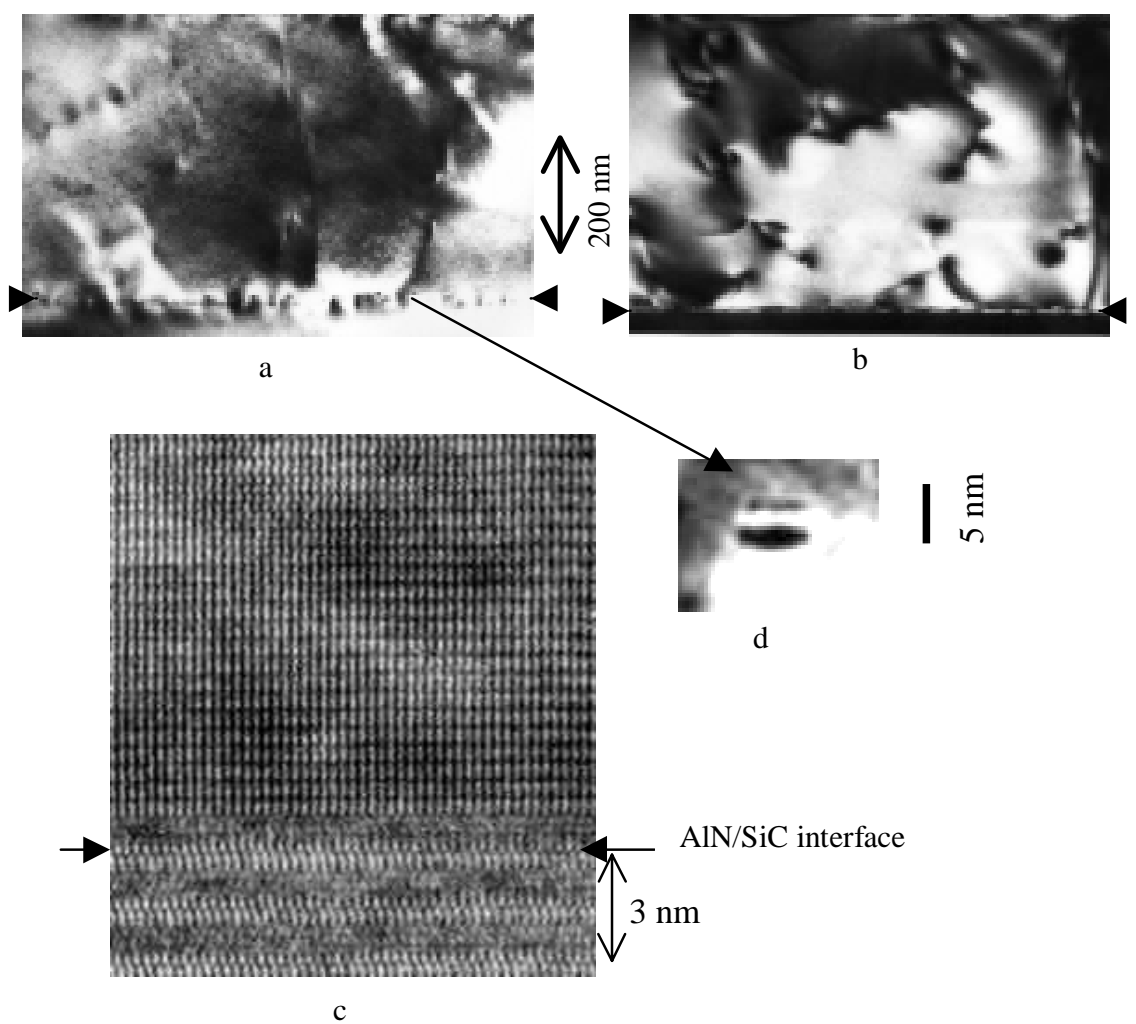

Fig. 2. (a) (0002) DF, (b) ( $01 \overline{1} 0$ ) DF and (c) high resolution lattice image of the bulk AlN/on-axis SiC interface. (d) Enlargement of defects seen just above the AlN/SiC interface in the (0002) DF image. 
showed small amounts of carbon incorporation. The small number and size of the defects discussed in the preceding paragraphs and their location at or near the AlN/SiC interface may preclude them from dissipating the surface charge.

SEM images of the samples' surfaces are shown in Figs. 3(a)-3(d). The surface of the sample grown on the off-axis seed (Figs. 3a-3b) has a step morphology with several cracks running perpendicular to the steps. There are several highly faceted large hexagonal grains, as shown in Fig. 3(b). The surface of the sample grown on the on-axis seed (Figs 3c-3d) contains many hexagonal grains stacked on top of one another. The surface is flatter than the surface of the AlN grown on the off-axis seed, and the grains are not faceted. Additionally, the top grains are much smaller than those seen on the AlN grown on the off-axis seed.
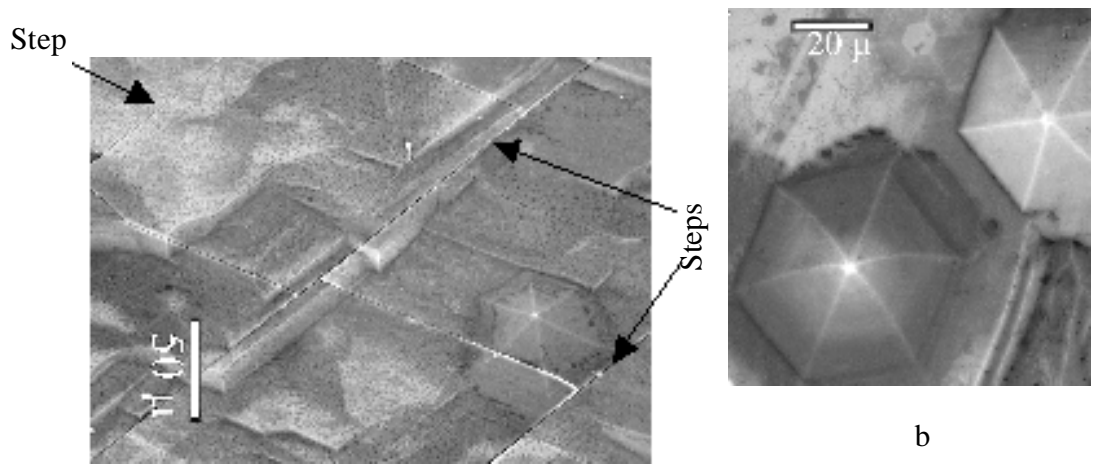

b

a
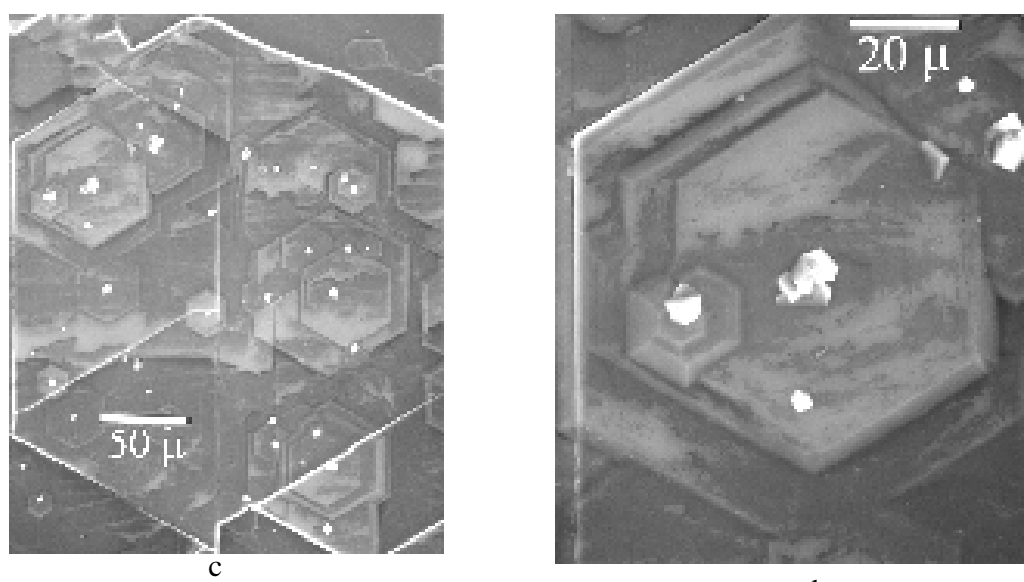

d

Fig. 3. SEM images of the samples grown on $3.5^{\circ}$ (a-b) off-axis $\mathrm{SiC}$, and (c-d) on-axis $\mathrm{SiC}$. The steps are labeled with arrows. 




$$
\stackrel{50}{\mathrm{~nm}} \quad \mathrm{a}
$$

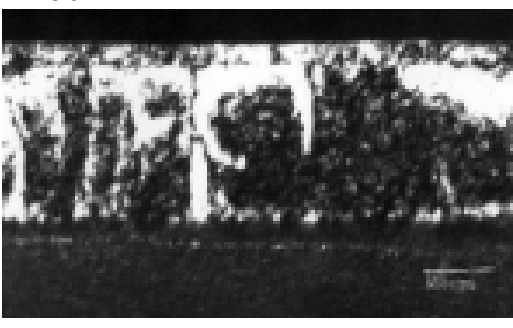

b

$\mathrm{X}$-ray results indicate that the AlN grown on on-axis $\mathrm{SiC}$ is of better quality than the AlN grown on off-axis AlN. Rocking curve measurements show that the full width half maximum (FWHM) of the (0002) peak for AlN grown on the on axis seed is 5.16 arc-minutes, while the FWHM of the AlN grown on the off-axis seed is 9.6 arc-minutes.

It is interesting to compare the morphology of the AlN grown by physical vapor transport to the AlN films we have grown by MOCVD. Figures 4(a) and 4(b) show (0002) DF images of AlN films grown on on-axis $\mathrm{SiC}$ and $3.5^{\circ}$ off-axis $\mathrm{SiC}$, respectively. Both films are highly columnar and contain an inversion domain boundary near the surface. These defects hinder the prospect of using these films as buffer layers for high quality nitride film growth.

\section{CONCLUSION}

Fig. 4. (0002) DF images of the AlN films grown on (a) $3.5^{\circ}$ off axis $\mathrm{SiC}$ and (b) on-axis $\mathrm{SiC}$ by MOCVD

The bulk AlN, particularly that grown on the on-axis $\mathrm{SiC}$, shows promising characteristics for the development of this material as a substrate. Further optimization of the growth conditions and a polishing technique to improve the surface quality is needed to yield an AlN surface suitable for high-quality nitride film growth.

\section{ACKNOWLEDGEMENTS}

The authors gratefully acknowledge the support of the MRCP Army Grant No. DAAL 019523530.

\section{REFERENCES}

[1] B.N. Sverdlov, G.A. Martin, and H. Morkoç, Appl. Phys. Lett. 67, 2063 (1995).

[2] Landolt and Börnstein, Numerical Data and Fundamental Relationships in Science and Technology, vol. 17, Semiconductors, Springer, Berlin (1984).

[3] G. Popovici, H. Morkoç, S.N. Mohammad, Group III Nitride Semiconductor Compounds, ed. B. Gil, Clarendon Press, Oxford, (1998). 